\title{
Can Digital Coaching Boost Your Performance? - A Qualitative Study among Physically Active People
}

\author{
Eeva Kettunen \\ University of Jyväskylä \\ eeva.k.kettunen@jyu.fi
}

\author{
Will Critchley \\ University of Jyväskylä \\ wcritchley@gmail.com
}

\author{
Tuomas Kari \\ University of Jyväskylä \\ tuomas.t.kari@jyu.fi
}

\begin{abstract}
The use of sport and wellness technology devices among athletes is highly popular. At the same time the demand for easy to understand, clear, and personalized information is also growing. Instead of numbers, users need and want solutions. Digital coaching can offer solutions for this by providing valuable training data and offering guidance and instructions on how to improve the training. This exploratory study focuses on the experiences, needs, and wants regarding a digital coach application among physically active people, more precisely crosscountry skiers. We found that the digital coach was perceived to have motivational elements. It was also viewed having potential to increase the awareness relating to personal performance level and technique as well as bring diversity into training. However, some perceived demotivating elements suggest that future development is needed. Our findings give insights to sport technology companies as well as athletes and coaches about the influence and possibilities of digital coaching among athletes and physically active people.
\end{abstract}

\section{Introduction}

Receiving relevant and reliable feedback is an important factor in improvement of physical activity and sports skills. Recent developments in technology, and particularly sport and wellness technology devices, have had a significant impact in the sports field. The information has enabled a new level of training feedback both during and after training sessions, which coaches and athletes consider valuable. The probability of learning increases when an athlete can compare the statistics of an actual movement with the internally expected optimum performance [1]. Therefore, the impact of information technology on physical activity and sport performance seems to be positive for helping athletes develop more effectively.
Feedback sources are considered more effective and relevant during the beginning of the skill acquisition process [2]. Their importance seems to decrease when the level of skill increases. Sport and wellness technology devices providing immediate feedback have been considered to be relevant for recreational, amateur, and professional athletes who are at an initial state of skill acquisition [1]. However, it is important to acknowledge that different people have different needs and wants regarding training feedback. Previous research has suggested that providing too much information, inadequate information, or unnecessary information might cause anxiety and stress to the users [3].

As sport and wellness technology devices become more developed and affordable, more athletes and other physically active people have integrated technology into their training. However, without professional help in interpreting the feedback, inappropriate adjustments in training may be made [4], which could lead to overtraining or even injuries. Therefore, sport and wellness technology companies should not only pay attention to the quality of the feedback but also ensure the information is easy to understand and implement. In previous studies it has been found that sport and wellness technology can increase motivation to exercise by increasing awareness of personal physical activity $[5,6,7,8]$. However, even if tracking physical activity data can lead to increased awareness, this effect might not be sufficient to maintain the use of sport and wellness technology [9]. Furthermore, this can have an effect on the maintenance of physical activity routines [10].

According to previous research $[11,12]$, users of sport and wellness technology prefer receiving relevant, clear, and easy-to-understand information from their devices and applications. They also appreciate receiving information on how to enhance or maintain their overall wellness and physical activity levels $[11,12,13]$. Receiving this type of data would likely lead to the use of sport and wellness technology being more goal-oriented [11,12], which can contribute 
to increase in motivation [14]. However, a typical problem with sport and wellness technologies is that they provide feedback through numbers and graphs instead of providing meaningful context and guidance. By providing personalized and more instructional feedback, such as personalized training plans, the devices could increase their users' motivation and goal-orientation towards improving physical activity and wellness and also make users more motivated to continue using the technology.

A prospective solution to serve this demand is digital coaching. Digital coaching refers to a "service on a technological device that not only gives feedback but also offers advice, suggestions and future steps for a user to follow in the pursuit of their wellness and fitness goals" [13, p.3]. The demand for digital coaching solutions to address goal-driven and personalized support towards physical activity goals was also recognized by Schmidt et al. [15]. While more traditional sport and wellness technology devices increase awareness based on physical activity feedback, digital coaching solutions are a step ahead. A digital coach can identify user's weaknesses and strengths and generate a personalized training plan for the user [15]. The use of digital coaching in a physical activity setting and its potential for increasing motivation has also been highlighted $[13,16,17]$.

The interest towards digital coaching solutions has increased among different stakeholders. However, because digital coaching solutions are still relatively novel, there has been few studies focusing on the usage experiences or the influence of digital coaching within users. To address this research gap, this study explores: 1. How can a sport and wellness technology digital coach influence physically active people when preparing for a race? and 2. What is the usage experience like and what are the perceptions, suggestions, and wishes regarding a digital coach in sport and wellness setting?. Physically active people are usually already familiar with and active users of sport and wellness technology. Having motivation to maintain physical activity level or improving their performance level, these people form an interesting target group for digital coaching solutions as well as for sport technology companies.

This study was conducted as a qualitative study in a cross-country skiing setting. The study included 25 athletes or otherwise physically active participants partaking in a ski marathon race. The study participants used a digital coach in preparation for the race. After the use period, the participants were interviewed to find insights, experiences, and suggestions for improvement regarding digital coaching. In addition, the theoretical focus was on studying the phenomenon from a motivational and psychological standpoint.

\section{Theoretical background}

The social cognitive theory by Bandura [18] is used as a theoretical framework for this study. This theory is widely used as a framework in studies regarding motivation and physical activity. Bandura also introduced the concept of self-efficacy as a part of this theory. Self-efficacy refers to a person's beliefs regarding his/her capabilities of performing a specific task. Whereas people with high self-efficacy are more likely to see difficult tasks as challenges and perform better, people with low self-efficacy might avoid doing a task they perceive as being too difficult [18]. There are four different sources of information that affect a person's self-efficacy: performance accomplishments, vicarious experience, verbal persuasion, and physiological states [18]. A person's self-efficacy is also related to his/her motivation; a task perceived as too difficult or too easy compared to the perceived skill level can decrease motivation. However, tasks that are perceived as moderately challenging can produce satisfaction by experiencing achievement [19].

Self-efficacy has an impact on enhancing sport performance and is a both a cause and effect of performance [20]. High self-efficacy has also been shown to accompany low pre-competition anxiety, high personal goals, strong goal importance, and high trait sport confidence [21]. Athletes with a high level of self-efficacy will also put more effort, persist longer, and also participate more frequently, enhancing their performance in sports [18]. Therefore, self-efficacy plays an important role in athletes' daily life.

When talking about motivation, the theory of selfdetermination [22] is also often discussed. The theory highlights three important components that affect motivation: the need for autonomy, the need for competence, and the need for relatedness. The need for autonomy refers to a person's need to be able to selfregulate their personal behavior. The need for competence refers to a person's need to be able to complete a given task by effectively interacting with the surrounding environment. The need for relatedness refers to a person's need to connect with others. All these components can individually or together facilitate intrinsic motivation. Intrinsic motivation means receiving satisfaction from doing an activity itself [22]. Related to physical activity and sports, having a high level of intrinsic motivation has often been connected with the formation of positive exercise habits as well as pursuing a more physically active lifestyle.

Another concept derived from social cognitive theory is proxy agency [23]. In this theory, people play an active and important role in their self-renewal, selfdevelopment, and adaptation by using mechanisms called "agencies". In this theoretical context, an 
"agency" means acts done on purpose. Bandura categorized three types of agents: personal agent, collective agent, and proxy agent. Personal agent means that the person him/herself acts as an agent, while the collective agent refers to a community or a group. Proxy agent refers to a situation where a third party acts as an agent on a person's behalf $[23,24]$. In a physical activity setting, a collective agent could be, for example, a team or a group and a proxy agent could be a coach or a personal trainer.

According to [23], there are three reasons why people use a proxy agent. First, people may feel they do not have the needed skills or knowledge to reach their desired goal. Second, they can perceive that a third person is more capable of facilitating the process towards the desired goal and outcome. Third, though people might have the needed skills and knowledge to pursue their desired outcome, they might want to transfer control of the process to another person since they do not want to have the responsibility of a direct control [25]. In a physical activity setting, using a proxy agent, such as a personal trainer, can help a person to manage environment and task demands as well as help in regulating and controlling exercise behavior. A proxy agent can also help in giving support in lifestyle management and in developing new skills [24]. A proxy agent may also be able to provide social support, which may increase the likelihood of full involvement, enjoyment, and focus [26]. In a sport setting, a proxy agent can influence an athlete's selfefficacy in different ways, such as providing vicarious experiences through examples or using persuasive techniques. Persuasive techniques, such as verbal persuasion, evaluative feedback, expectations, and imagery are widely used by trainers and coaches [21].

In this study, the proxy agency theory is examined from a digital coach point of view, and the concept of proxy agent refers to a sport and wellness technology device or application, which is combined with digital coaching features. Like a human proxy agent, a digital coach can also influence feelings of competence by providing feedback, instructions, and verbal persuasion as well as gathering and showing data about performance accomplishments. A digital coach can also affect feelings of relatedness by providing a tool for social comparison. However, social comparison provided by sport and wellness technology can also negatively affect intrinsic motivation by decreasing feelings of perceived competence [27].

It has been suggested that long term use of a proxy agent can lower the self-regulatory skills of a user which are necessary for independent management of sport and physical activity participation [28]. Compared to using a human proxy agent, a digital coach as a proxy agent requires a certain amount of independence and regulatory skills and therefore encourages the user to practice independence while still in a proxy content. This affects positively the feeling of autonomy. However, since using a digital coach, rather than a human coach, gives more responsibility for the user, the concerns regarding appropriate interpretation and delivery become more present.

\section{Methodology}

\subsection{The digital coach used in this study}

The digital coach used in this study was Racefox Ski [29]. Racefox uses a smartphone application and an ECG heart rate monitor style chest belt. The belt is also mounted with an accelerometer pod. The data collected by the pod is transferred to a smartphone in real-time via Bluetooth. The application provides data in realtime on the screen and may also send information to a person's connected headphones [29].

The application provides technique analysis and feedback, training program creation, and real-time exercise monitoring and instruction. The features enable the application to analyze a user's technique, provide objective, measurable feedback on their technique, provide specific feedback on how to improve a user's technique, and automatically generate training programs based on the user's fitness goals.

The technique analysis is performed initially using a timed technique test. The skier's technique is measured with the accelerometer and analyzed in four key performance indices (KPI). The optimal technique values for these KPIs are based on analysis of worldclass Norwegian skiers. Distance and heart rate may also be measured. The application displays the KPIs data from the test and a brief text with feedback on the user's technique and some advice on how to improve.

Based on the test data, and optionally the user's goal time for a specific race, a two-week training program can be created, consisting of specific guided interval sessions supplemented with easy skiing sessions. After two weeks, the technique test is performed again, and a new program is created based on the new results. The interval sessions instruct the user to reach a specific value in one of the four KPIs. When a user follows one of the guided workout sessions, they are provided real-time feedback, through headphones or their smartphone's speakers, on whether they are achieving the KPI value during the session. For example, a "positive" or "negative" tone will be made for each double poling stroke the skier makes, based on whether or not they have reached the target KPI. After the session, the application will provide 
feedback on the skier's technique and how successful they were in achieving the goals of the workout.

\subsection{Data collection and analysis}

This study followed a qualitative approach. The target population of the study was physically active cross-country skiers (henceforth referred to as skiers) who participated in a ski marathon race that took place in Finland on March 2018. As the digital coach used in this study is designed for cross-country skiing, skiers were an obvious choice for the target group. The device itself was one of the most advanced digital coaching solutions in the market focusing on improving sport performance. Because aim was also to investigate the digital coach as a race preparation tool, the participants were recruited from race participants.

The race had two optional distances of $30 \mathrm{~km}$ or $50 \mathrm{~km}$ which both could be skied in skate or classic style. The study participants were recruited with the help of the ski club that organized the race. The invitation to participate in the study was sent via email by the ski club to approximately 900 skiers already registered for the race. A total of 40 people expressed their interest to take part in the study. Out of these, 25 were doing the race in a classic style and expressed their interest for using a digital coach in training for the race. Since the Racefox digital coach was (at the time of the study) only able to give feedback and instructions regarding classic style skiing, the skiing technique was used as a criterion in participant recruitment. Therefore, the number of participants recruited for the study was 25 . However, after the use period, two participants reported having problems using Racefox, and therefore the data collected from them was limited to future suggestions regarding digital coaching whereas the parts regarding user experiences and race preparation were left unanswered.

The age of the participants ranged from 21 to 74 years. The participant group consisted of an almost equal number of male and female participants. Most participants in the study were in the $50 \mathrm{~km}$ race. The skiing experience level of the participants ranged from current or previous competitive skiers to participants who were doing a long-distance ski race for the first time. All the participants were physically very active regardless of their skiing experience. The information regarding the participants physical activity level was collected by using a scale based on Finnish National Sport Survey [30] and consisted of seven categories. The categories presented in the order from the most active to the least active were: competition athletes, fitness athletes, fitness participants, health enhancing participants, utilitarian participants, casual participants, and inactive or sedentary people. Only one participant had previous experiences regarding digital coaching. All participants had some experience with sport technology and most were using solutions like heart rate monitors or physical activity applications in their everyday training. Table 1 presents more detailed information about the study participants.

Table 1. Sample description

\begin{tabular}{|l|c|c|}
\hline & \multicolumn{2}{|c|}{ Participants (N = 25) } \\
\hline Gender & $\mathrm{n}$ & $\%$ \\
\hline Male & 12 & 48 \\
\hline Female & 13 & 52 \\
\hline Age & & \\
\hline $21-30$ years & 3 & 12 \\
\hline $31-40$ years & 6 & 24 \\
\hline $41-50$ years & 9 & 36 \\
\hline $51-60$ years & 5 & 20 \\
\hline $61-70$ years & 1 & 4 \\
\hline $71-80$ years & 1 & 4 \\
\hline Skiing distance & & \\
\hline 30 km & 2 & 8 \\
\hline 50 km & 23 & 92 \\
\hline Physical activity level & & \\
\hline Competition athletes & 4 & 16 \\
\hline Fitness athletes & 14 & 56 \\
\hline Fitness participants & 7 & 28 \\
\hline
\end{tabular}

All 25 study participants were given a personal Racefox Ski digital coach and were asked to use it in the manner that is most suitable to them. The use period started approximately one month before the ski marathon race and lasted approximately eight weeks. During the use period, data was collected two times. First, all the participants were sent an online survey four days before the race where they were asked questions regarding preparation for the upcoming ski marathon with the digital coach and also about their overall feelings regarding the upcoming ski race. The survey questionnaire was created based on previous literature and the theoretical background with the aim to provide relevant knowledge to answer our research questions. All the participants were asked to answer the survey before the race so that their answers would not be affected by the race results. The second round of data collection took part two to three weeks after the race. This time all the participants were interviewed using a thematic semi-structured interview method. 
The interviews were performed over skype, by phone, or in person and lasted 55 minutes on average.

The data was analyzed using thematic analysis which is the most widely used analysis method for qualitative research [31]. Thematic analysis is a method for "identifying, analyzing and reporting patterns (themes) within data" [32, p.79]. It allows describing the data set with rich detail. The guidelines by Braun and Clarke [32] were followed when doing the data analysis process and as recommended, the guidelines were adjusted in order to fit the research aim of the study [32]. The data analysis started by getting familiar with the data and transcribing the relevant parts of the interviews. In the interview phase, the data was already divided into three different parts which were: 1 . user experiences, 2 . race preparation with a digital coach, and 3. ideal digital coach. All of these themes were individually studied aiming at finding differences, similarities, and commonalities in the responses. Finally, the report was created, which aimed to highlight all the aspects that were considered important by the participants regarding each theme.

\section{Results}

This section introduces the key aspects participants considered important regarding digital coaching. The results are presented in three themes starting from user experiences, followed by race preparation, and finally focusing on the ideas and recommendations the participants had on digital coaching in general.

\subsection{User experiences}

Almost all the participants reported the initial implementation of the product to be easy. The most often used features in Racefox (henceforth referred to as device) were technique tests and distance training, which were used by almost all participants. More than half of the participants followed the training plan given by the device or at least adapted some recommended training sessions to their own existing training plan. Some participants were not able to or did not want to follow the training plan. Example reasons for this were that the training plan was not suitable and not taking into account the other parts of life and busy schedules. Some participants also highlighted that the training plan could not be adapted based on health reasons, for example when getting a flu during the training period.

A few participants called themselves "performers", meaning they get excited if someone or something gives them a specific plan which they can then follow precisely: "Training is not goal oriented without a training plan. Having a plan brings a different kind of focus on the training". Whereas these "performers" followed the training program received from their digital coach, other participants decided to stick with their old training routine and then see if the digital coach could somehow bring some tips on how they could make their own program more versatile: "Devices are devices and I decide what I do. If I agree with the device, only then I can do what it says". As one participant highlighted, "training with a new coach requires a conscious decision to be willing to let the coach guide one's training".

Around half of the participants tried the real-time feedback function during their training. For all of them this was a feature they had never tried before and it was perceived as a positive and useful feature: "Realtime feedback really helped me to understand the exact moments when I have to do something differently".

All participants were interested in the feedback received from the device. One third of the participants felt the quality of the feedback was high. They thought the feedback was well presented and easy to understand. In particular, the graphical presentation style of feedback was perceived useful. The feedback made them think about their skiing and technique from a different perspective from what they were used to.

Regardless of positive experiences regarding feedback, most of the participants would have liked the feedback to be presented more clearly and felt it was difficult to relate the feedback to their own training and technique. Therefore, they would have liked to be able to ask more specific questions in order to increase their understanding. However, some participants explained that also their level of understanding of what is "good" skiing technique increased the more they used the device. Some participants wanted more detailed feedback since they felt the received feedback was often too similar. Some participants commented that the device was not able to take into account the level of tiredness nor the aim of the ski, meaning whether the ski was meant to be a high-intensity training or just a recovery ski. Therefore, the device was not able to adjust the feedback to the purpose of the training.

The participants were asked about the most positive experiences they had regarding the usage. The most highlighted point was that the digital coach was able to make the training more versatile, for example by offering various interval trainings, which many participants reported they rarely do: "I did different trainings and it made me push really hard which motivated me a lot. It also gave me suggestions for the following training". Participants also appreciated receiving different kinds of data from their skiing, which made them pay more attention to their technique overall. Another highly appreciated feature was the individualized real-time feedback based on the data 
from the technique tests. From a technical standpoint, the participants appreciated that the device was quick and easy to start in the beginning of a training session.

Besides positive experiences, participants also shared their negative ones. The biggest negative experiences were related to the functionality of the device. Participants did not always enjoy having to use their phone in cold weather, since taking off their gloves just before and after skiing was somewhat annoying. Issues related to reliability were also often mentioned. Sometimes training sessions were not recorded due to cold weather or loss of the Bluetooth or GPS signal. Some users said that these negative issues made the skiing experience less enjoyable. Some participants said that their motivation for using the device was lowered by the similarity of the received feedback, and that they would have wanted to receive more practical personalized instructions: "I always received the same feedback. It made me feel I was not able to change my skiing style at all". Another remark a few participants made concerned the relationship of the feedback to their own skill level. They wished having a chance to determine own skill level beforehand and based on that, receive feedback adjusted to other skiers with similar skill level instead of being compared to professional skiers. This would require a databank consisting with technique and performance values for different levels of skiers.

In the end of the use period, participants were also asked about their trust towards the digital coach in contrast to other sport technology devices they have used before. Majority of the participants expressed their trust on the actual data received from the digital coach. The trust towards the functionality of the device was also relatively high. Some participants expressed that they trusted the data even more than a feedback received from an actual person. However, the lack of overall knowledge related to the source of the "ideal technique" data as well as having a too complex operating system were reasons that created distrust. Despite perceiving the data as reliable, some participants highlighted the importance of common sense: "You should always have a filter on when reading information received from a device".

When asked about digital coaching in general, the participants perceived that the use of a digital coach can positively affect their exercise motivation. The most important factor for increasing motivation seemed to be the ability to monitor performance, receive feedback, and see development over time. Another motivational factor was the possibility to have more variety in training by receiving training advice and suggestions. A third motivational element was having the feeling that someone is always watching and recording training and provides encouragement on a regular basis. Besides the positive factors, the participants also experienced some demotivating elements. If the device did not work properly, training enjoyment could decrease since the focus is more on technical issues rather than actual performance. Some participants perceived that having a constant reminder and observer might create stress to some users. A digital coach might also turn the focus too much on performance improvement, which might lead to overtraining. As one participant expressed, having a right mindset is important: "The device does not tell me what to do, it is me who makes the decisions".

\subsection{Preparing for a race}

Since the first month of the use period occurred while the participants were preparing for the race, they were also asked how using digital coaching affected their race preparation. Most participants reported having had a slightly different race preparation from using of digital coach. The device encouraged them to do more interval training instead of familiar longer steady paced trainings. Around two thirds of the participants reported to have made changes to their race preparation routines. These changes included more training sessions, more physically intensive trainings, or more specific types of technique trainings. Using the digital coach helped several participants learn more about skiing technique in general and helped them pay more attention to skiing technique. Some participants reported having been more focused during training sessions. In particular, exercises related to double poling were utilized by many participants. Some mentioned seeing development in their double poling technique, which gave them confidence for the race since the last part of the course was suitable for double poling technique. One of the participants also felt a confidence boost during the race: "In the race I felt that unlike previous years, I was more confident in my skiing ability when comparing myself to others. My technique was better due to Racefox. A coach would not have been able to give me this information".

Using a digital coach during race preparation seemed to increase the motivation to train for some participants by providing encouragement and offering ready-made exercises. Some participants reported that technical usability problems decreased their motivation over time or even made training feel negative.

Participants were also asked about they thought using a digital coach had affected their self-efficacy regarding the race. Slightly less than half reported feeling more confident about the race from using the digital coach. This increased confidence came from perceived improvement of skiing technique, received assurance of the level of one's own skiing technique, 
the assurance of being able to change the skiing technique according to different conditions and the positive verbal persuasion received from the device. One participant stated: "I received more confidence when I heard 'You are a good skier'. I knew that, but it was nice to hear it from somebody else". Only one participant reported a negative influence on confidence for the race due to the digital coach: "The trainings felt hard. I got a feeling that how can I ever survive the race if I am not even able to survive these trainings".

Regarding race preparation, several participants stated that it would have been better to use the digital coach for longer than only one month before the race. Due to the short timeframe, some participants wanted to stick to their own familiar training routine and used the digital coach only to bring extra flavor to the training. Participants highlighted that having the coach for a longer time would have provided a better chance to see the actual development, which might have influenced their self-efficacy even more. For one participant having not enough time before the race had negative results: "Too short time caused stress. You have to allocate time for learning to use the device."

Finally, participants were asked how essential and important they see the use of a digital coach when preparing for a race. Around one third did not see the role of a digital coach as notably important and said they would rely more on personal experience and personal feeling as well as already established race preparation routines. A bit less than one third considered using a digital coach important in race preparation by giving tips in improving technique, providing a training plan, giving guidance regarding race preparation, and increasing motivation to train. They said they would be willing to pay for the product in its current form. The rest appreciated the benefits and the potential of a device like Racefox, but did not necessarily see the device, in its current form, as being essential. In general, the participants felt that the two main target groups who would benefit most from using a digital coach in race preparation would be people with less experience and people who want to compete and are aiming at improving their performance.

\subsection{The ideal digital coach}

After testing the digital coach device for approximately eight weeks, the participants had some idea what digital coaching can be like. After the experience, the participants were asked to describe their ideal digital coach. The most recommended option was a watch combined with an optical heart rate sensor or a heart rate belt. The most important factor highlighted regarding usability was that the device should be as light and unobtrusive as possible and easy to operate mid-exercise. Therefore, most people felt a phone application to be sub-optimal since they prefer to not carry their phone while exercising. For some, even wearing a chest belt felt too oppressive. Some participants said they would like to see the exercise data from a computer and suggested an automatic data transfer from the digital coach to a computer.

Since the importance of high quality and accurate feedback had stood out already regarding user experiences, the participants had suggestions on how their ideal digital coach would give feedback. The most preferred way of receiving feedback during the training was via audio. However, post-training, participants wished to have text feedback which could be seen both from a computer and the device. Some participants also wished additional simplified feedback represented with numbers, graphics, or even emojis. However, this feedback would also need to be paired with more indepth explanations and suggestions expressed in text form. Overall, personalized and accurate feedback was considered important combined with easy to understand personalized instructions.

The most important reason for participants to use a digital coach was to be able to improve their performance. This could be done by making training more systematic and planned and by providing ways to increase the overall knowledge of training and person's individual characteristics. Besides technique, training, and performance related feedback, some participants also wanted recovery related feedback from their ideal digital coach. Recovery related feedback would include tips for nutrition, hydration, and rest. However, general advice or instructions regarding diet, nutrition, or sleep were not considered necessary by most of the participants since these were considered being part of the knowledge they already had. Only a few were interested in including daily activity monitoring for their ideal coach. One participant suggested adding imagery related mental training exercises.

Regarding having the right attitude, one participant made a development proposal: "The devices should have different personalities, such as encouraging or demanding, designed for different types of people. The devices could be branded with personas and use those in marketing."

Finally, participants gave their preferences and perceptions when comparing a digital coach to a human coach. Just over half reported they would use a digital coach over a human coach in their current situation, while less than one third preferred a human coach. The rest wished they could use a combination of human and digital coach. The reason for choosing a digital coach was to be independent in scheduling training, since the digital coach would always be available. The digital coach was also seen as a more 
affordable option. Some participants perceived a digital coach having a larger and more science-based knowledge, which makes the information more reliable. Also, receiving constant real-time feedback was perceived as an advantage. While the information received from a digital coach was perceived as more objective, human coaches were considered advantageous in their ability to have a more holistic view of the person. This enables the coach to make changes to the training program based on factors such as life outside training, emotional states, or physical injuries. A human coach can also provide more information and instructions on request, and through personal interaction, motivate each individual in a way that is most suitable and effective for them.

\section{Discussion}

The purpose of this study was to explore physically active people's experiences regarding digital coaching by focusing on the following questions: 1 . How can a sport and wellness technology digital coach influence physically active people when preparing for a race? and 2. What is the usage experience like and what are the perceptions, suggestions, and wishes regarding a digital coach in sport and wellness setting?

The findings indicate that physically active people are interested in digital coaching as a way to improve their sport performance. Digital coaching solutions are generally perceived as interesting, possessing potential to enhance the quality of training and performance by focusing on personalized feedback and guidance. On average, participants found using a digital coach motivating, supporting previous research [16,17], but also negative motivating factors were found.

A digital coach can be a useful tool in race preparation. It can change normal training routines by adding more specific, intensive, and somewhat more efficient trainings. While most participants perceived this positive and motivational, a change in routines can also be seen stressful especially if there is not enough time to adjust. Following a personalized training program can also help in getting into better physical shape. Receiving personalized feedback can increase the awareness of technique, which leads to higher focus on trainings. In particular, real-time feedback was perceived as helpful. Seeing improvements in technique can also increase confidence regarding an upcoming race. This suggests that the increased awareness of personal technique and the feedback of improvement can positively affect self-efficacy regarding an upcoming race, supporting the findings of [33]. Therefore, it can be concluded that a digital coach is able to affect the level of self-efficacy through showing data of performance accomplishments and providing verbal persuasion.

In terms of the proxy agency theory, the biggest reason why physically active people would want a digital coach is that they perceive that a digital coach can bring extra knowledge and instructions and therefore is more capable of facilitating the process towards the desired goal and outcomes. Another reason physically active people can be interested in digital coaching would be the possibility to give responsibility of planning the training to somebody else. This would give extra time within their schedule as well as reduce the level of stress. For some people having being told what to do can even bring extra motivation. On the other hand, the reasons some people are not excited about digital coaching seem to relate to already having a large amount of information acquired from many years of experience or having little desire to improve their performance level.

The biggest reasons why physically active people would prefer their personal trainer to be a sport and wellness technology device rather than a real person, is that the devices are perceived as easy, flexible, and affordable. The users did not want to be dependent on anyone else, and a device can be used whenever needed. Also, the objective, accurate, and comparable information based on scientific background is seen as an advantage. However, the lack of human elements, such as the ability to communicate and detect feelings, or to understand the relationship between training and personal life seems to decrease the attractiveness.

From the self-determination theory standpoint, these results suggest that a digital coach can be seen motivational by increasing feelings of autonomy. By providing an opportunity to see personal development through objective data, a digital coach can also affect the feeling of perceived competence. However, based on the results, a digital coach can also have negative effects on motivation, for example, if the feeling of competence is lowered due negative or unclear feedback from the digital coach.

As a practical contribution, we present implications for future development of digital coaches. A key issue is to further increase the level of personalized feedback and instructions while integrating human elements into the digital coaching solutions. For example, users could be given an opportunity to personalize the style of feedback to better match their personality. Improvements related to usability and personalized feedback might further increase the positive motivational affects and diminish the negative ones.

Based on the findings, the experiences, wants, and needs regarding digital coaching vary between different people. However, the two main target groups seen as most potential for digital coaching are people 
with lower sports skill levels and people who, despite already being skillful and competitive, want to further improve their performance. For physically active people the usability factors also seem to play an important role. It is essential that the device is unnoticeable and does not hinder the sport activity. It is also important that the digital coach is easy to use during the training and that the use does not cause interruptions or make the training feel less enjoyable.

Professionals working on sport and physical activity settings could also benefit from integrating digital coaching solutions in their interventions. Yardley et al. [34] highlighted that it is important, especially in digital interventions, to have a product that uses a person-based approach while also having an emphasis on independent use and autonomy. This is considered essential in maximizing the effectiveness and acceptability of interventions [34]. Based on our findings, a combination of a digital coach and a human coach is recommendable. A human coach can help in analyzing the results received from the digital coach, and the device can also give the coach more in-depth knowledge about the user. This makes personal trainers a potential target group for digital coaching products.

\section{Limitations and future research}

This study has a few notable limitations. First, the use period was approximately eight weeks, including only one-month time for race preparation. This limits the findings to a relatively short-term experience. However, the use period was long enough for the participants to get an idea about the capabilities of having a digital coach as a trainer. Regardless, future studies could be done with longer use periods. Second, the digital coach used in this study was specifically designed for cross-country skiing. Thus, it should not be assumed that these results are directly applicable to other types of digital coaching solutions that may focus on other types of feedback and assistance or are meant for different target groups. Also, because the level of a person's cross-country skiing technique plays such a major role in performance, the results of this study may also not be directly comparable to other sports.

Regardless of these limitations, using a digital coach encouraged the participants to think about the concept of digital coaching in general which helped them to express their needs, motives, and wants related to this topic. The findings regarding user motives and suggestions for product development can be applied to other devices targeted for these types of people. This study is among the first to study the topic of digital coaching with athletes and physically active people, and more research in this field should be done. Future studies could focus on measuring the actual effects sport and wellness related digital coaching has on sport performance, motivation, or self-efficacy within different target groups. Since the results revealed also some elements affecting motivation negatively, special focus in future studies could be on these elements. Overall, the topic of digital coaching within people of any level of physical activity continues to be an important topic of research.

\section{References}

[1] D. Liebermann, L. Katz, M. Hughes, R. Bartlett, J. McClements and I. Franks, "Advances in the Application of Information Technology to Sport Performance", Journal of Sports Sciences, 20(10), 2002, pp. 755-769.

[2] C. Winstein and R. Schmidt, "Reduced Frequency of Knowledge of Results Enhances Motor Skill Learning", Journal of Experimental Psychology: Learning, Memory, and Cognition, 16, 1990, pp. 677-691.

[3] S. Halson, J. Peake and J. Sullivan, "Wearable Technology for athletes: Information Overload and Pseudoscience?" International Journal of Sports Physiology and Performance, 11(6), 2016, 705-706.

[4] P. Düking, A. Hotho, H-C. Holmberg, F. Fuss and B. Sperlich, "Comparison of Non-invasive Individual Monitoring of the Training and Health of Athletes with Commercially Available Wearable Technologies." Frontiers in Physiology, 7 (71), 2016, pp. 1-11.

[5] M. Kang, S.J. Marshall, T.V. Barreira and J. O. Lee, "Effect of Pedometer-based Physical Activity Interventions: a Meta-analysis" Research Quarterly for Exercise and Sport, 80(3), 2009, pp. 648-655.

[6] T. Kari, T. J. Piippo, L. Frank, M. Makkonen and P. Moilanen, "To gamify or not to gamify?: Gamification in Exercise Applications and Its Role in Impacting Exercise Motivation", Proceedings of the 29th Bled eConference, Bled, Slovenia, University of Maribor, 2016b, pp. 393-405.

[7] T. Kari, E. Kettunen, P. Moilanen and L. Frank, "Wellness Technology Use in Everyday Life: A Diary Study", Proceedings of the 30th Bled eConference: Bled, Slovenia, University of Maribor, 2017a, pp. 279-294.

[8] J.B. Wang, J.K. Cataldo, G.X. Ayala, L. Natarajan, L.A. Cadmus-Bertram, M.M. White... and J.P. Pierce, "Mobile and Wearable Device Features that Matter in Promoting Physical Activity", Journal of Mobile Technology in Medicine, 5(2), 2016, pp. 2-11.

[9] S.W. Miyamoto, S. Henderson, H.M. Young, A. Pande and J.J. Han, (2016). "Tracking Health Data is Not Enough: a Qualitative Exploration of the Role of Healthcare Partnerships and mHealth Technology to Promote Physical 
Activity and to Sustain Behavior Change", JMIR mHealth and uHealth 4(1), 2016, pp. e5.

[10] M.U. Warraich, "Wellness Routines with Wearable Activity Trackers: A Systematic Review", Proceedings of the 10th Mediterranean Conference on Information Systems (MCIS), Paphos, Cyprus: AIS, 2016.

[11] T. Kari, S. Koivunen, L. Frank, M. Makkonen and P. Moilanen, "Critical Experiences During the Implementation of a Self-Tracking Technology", Proceedings of the 20th Pacific Asia Conference on Information Systems (PACIS), Chiayi, Taiwan: AIS, 2016a.

[12] T. Kari, S. Koivunen, L. Frank, M. Makkonen and P. Moilanen, "The Expected and Perceived Well-being Effects of Short-term Self-tracking Technology Use", International Journal of Networking and Virtual Organisations, 17(4), 2017b, pp. 354-370.

[13] E. Kettunen and T Kari. "Can Sport and Wellness Technology be My Personal Trainer? - Teenagers and Digital Coaching", Proceedings of the 30th Bled eConference, Bled, Slovenia, 2018.

[14] M. K. Shilts, M. Horowitz and M.S. Townsend, "Goal Setting as a Strategy for Dietary and Physical Activity Behavior Change: a Review of the Literature", American Journal of Health Promotion, 19(2), 2004, pp. 81-93.

[15] B. Schmidt, S. Benchea, R. Eichin and C. Meurisch, "Fitness Tracker or Digital Personal Coach: How to Personalize Training." Proceedings of the ACM International Joint Conference on Pervasive and Ubiquitous Computing and ACM International Symposium on Wearable Computers, Osaka, Japan: ACM, 2015, pp. 1063-1067.

[16] M. Kranz, A. Möller, N. Hammerla, S. Diewald, L. Roalter, T. Ploetz and P. Olivier, "The Mobile Fitness Coach: Towards Individualized Skill Assessment Using Personalized Mobile Devices" Pervasive and Mobile Computing 9, 2013, pp. 203-215.

[17] T. Kari and P. Rinne. "Influence of Digital Coaching on Physical Activity: Motivation and Behaviour of Physically Inactive Individuals", Proceedings of the 31st Bled eConference, Bled, Slovenia, 2018.

[18] Bandura, A., Social Foundations of Thought and Action: A Social Cognitive Theory, Englewood Cliffs, NJ: PrenticeHall, 1986.

[19] A. Bandura, "Health promotion from the Perspective of Social Cognitive Theory", Psychology and Health, 13(4), 1998, pp. 623-649.

[20] S. Moritz, D. Feltz, K. Fahrbach, and D. Mack, "The Relation of Self-efficacy Measures to Sport Performance: A Meta-analytic Review", Research Quarterly for Exercise and Sport, 71(3), 2000, pp. 280-294.
[21] D. Feltz and C. Lirgg, (2001). "Self-efficacy Beliefs of Athletes, Team, and Coaches" In R. Singer, H. Hausenblas and C. Janelle (Eds.) Handbook of Sport Psychology, 2nd ed., New York: John Wiley \& Sons, 2001, pp. 340-361.

[22] R.M. Ryan and E.L. Deci, "Intrinsic and Extrinsic Motivations: Classic Definitions and New Directions", Contemporary Educational Psychology, 25(1), 2000, pp. 5467.

[23] A. Bandura, "Self-efficacy Mechanism in Human Agency”, American Psychologist, 37, 1982, pp. 122-147.

[24] Beauchamp, M. R. and M. A. Eys, Group Dynamics in Exercise and Sport Psychology - Contemporary Themes, New York, NY: Routledge, 2007.

[25] Bandura, A., Self-efficacy: The Exercise of Control. New York, NY: Freeman, 1997.

[26] Jowett, S. and D. Lavallee, Social Psychology in Sport. Champaign, IL: Human Kinetics, 2007.

[27] C. Kerner and V. Goodyear. "The Motivational Impact of Wearable Healthy Lifestyle Technologies: A Selfdetermination Prospective on Fitbits With Adolescents", American Journal of Health Education, 48(5), 2017, pp. 287297

[28] C. Shields and L. Brawley, "Preferring Proxy-agency: Impact on Self-efficacy for Exercise", Journal of Health Psychology, 11(6), 2006, pp. 904-914.

[29] Racefox, Racefox Ski, 2018, URL: https://racefox.se/ski\#!sv-ski. (visited on 05/29/2018).

[30] Finnish Sports Federation, Kansallinen Liikuntatutkimus 2009-2010: Aikuis- ja senioriliikunta. [National Sports Survey 2009-2010] Report, Finnish Sports Federation, Helsinki, Finland, 2011.

[31] Guest, G., K.M. MacQueen and E.E. Namey, Applied Thematic Analysis. Los Angeles, CA: SAGE, 2012.

[32] V. Braun, and V. Clarke, "Using Thematic Analysis in Psychology", Qualitative Research in Psychology, 3(2), 2006, pp. 77-101.

[33] E. Kettunen, T. Kari, M, Makkonen and W. Critchley. "Digital Coaching And Athlete's Self-Efficacy - A Quantitative Study on Sport and Wellness Technology", Proceedings of the 12th Mediterranean Conference on Information Systems, Corfu, Greece, 2018.

[34] L. Yardley, L. Morrison, K. Bradbury and I. Muller, "The Person-Based Approach to Intervention Development: Application to Digital Health-Related Behavior Change Interventions", Journal of Medical Internet Research, 17(1), 2015, pp. e30. 